\title{
DESIGN, FABRICATION AND CHARA- CTERIZATION OF A FLEXIBLE SYSTEM BASED ON THERMAL GLUE FOR IN AIR AND IN SEM MICROASSEMBLY
}

\author{
Cédric Clévy ${ }^{1}$, Arnaud Hubert $^{1}$, Stephan Fahlbusch ${ }^{2}$, Nicolas Chaillet ${ }^{1}$ \\ and Johann Michler ${ }^{2}$ \\ ${ }^{1}$ Laboratoire d'Automatique de Besançon -UMR CNRS 6596 - ENSMM - UFC \\ 24 rue Alain Savary, 25000 Besançon, FRANCE \\ ${ }_{2}^{2}$ Swiss Federal Laboratories for Materials Testing and Research (EMPA), \\ Feuerwerkerstrasse 39, 3602 Thun, SWITZERLAND
}

\begin{abstract}
This paper presents the design, fabrication and characterization of a device able to exchange the tip part (so-called the tools) of a two fingered microgripper. The principle of this tool changer is based on the use of a thermal glue whose state (liquid or solid) is changed by heating or cooling. Several kinds of pairs of tools have been designed. The suitable pair of tools can be chosen according to the size, shape and material of the object to manipulate. The tool changer enables one to perform a sequence of elementary micromanipulation tasks (i.e. an assembly sequence) by using only one gripper mounted on only one manipulator. The tool changer has been automated and successfully tested in air and in the vacuum chamber of a Scanning Electron Microscope (SEM). It brings flexibility to the micromanipulation cell and contributes to reduce the costs, the used space and experimentations time for micromanipulations in the SEM. The assembly of a ball bearing (the balls are $200 \mu \mathrm{m}$ in diameter) has been successfully tested using the microgripper equipped with the tool changer in a SEM. This tool changer has been designed for a microgripper but can be easily adapted to lots of other kinds of systems.
\end{abstract}

Keywords: Micromanipulation cell, Tool Changer, Micromanipulation, Microassembly, Flexibility, Microfactory, Scanning Electron Microscope, Automation.

\section{Introduction}

Great developments have been done in the field of microrobotics for the past few years. Several very efficient actuators have been developed 


\section{Introduction}

Great developments have been done in the field of microrobotics for the past few years. Several very efficient actuators have been developed to be used in microassembly workcells ([3] [1]). Nevertheless, in the field of micromanipulation, two main challenging topics are still growing.

First of all, some research works are done to automate microassembly cells ([7]) ([18]). Very small and precise sensors must be developed and a lot of work is also done to integrate vision capabilities ([8]) ([17]). These researches may lead toward the development of microfactories ([2]) ([15]).

Secondly, performing micromanipulation tasks inside the chamber of a Scanning Electron Microscope (SEM) is very useful. Indeed, the large depth of focus, the high magnification and the clean enviromment of a SEM provide very good conditions to micromechanical studies or to microassembly. These researches led toward the development of very compact and SEM compatible devices ([11]) ([14]).

These application fields require flexible micromanipulation cells. To perform micromanipulation tasks with flexibility, we have designed a microgripper and its tool changer. Up to now, few works have been done to bring fexibility ([10]) ([9]) ([2]]). Several systems have been developed but are closer to a miniaturization approach ([11]) ([19]) using for example revolver turrets ([20]) ([6]). Few devices are adapted to the microworld. They allow temporary mechanical fixation ([16]) ([12]). Our solution was developed to close a part of this gap. The principle of our tool changer will be presented in section 2 and its characterization will be studied in section 3 . Finally, this system will be used to perform the assembly of a ball bearing in a SEM (section 4). 


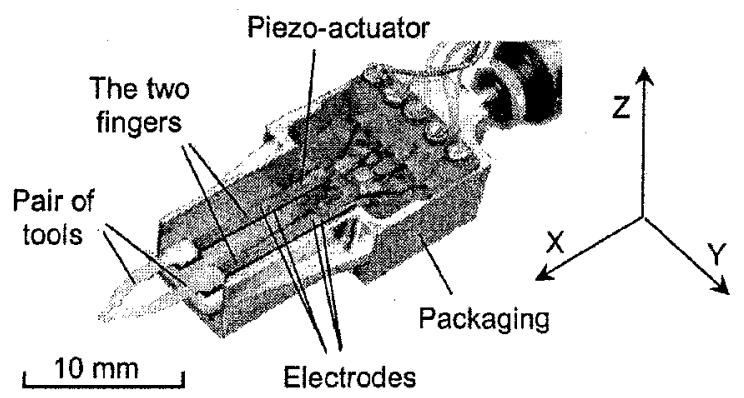

Figure 1: Overview of the microgripper.
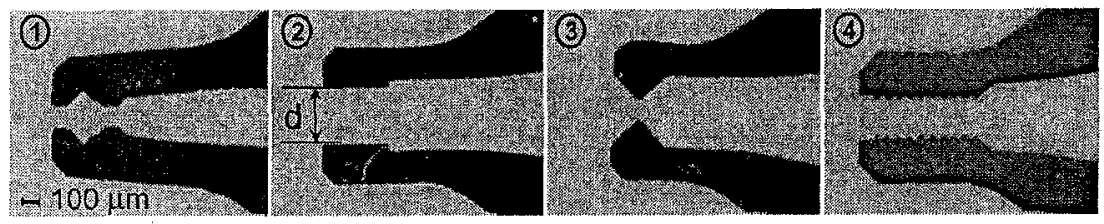

Figure 2: Different kinds of pair of available tools. These tools are made of Nickel, are $200 \mu \mathrm{m}$ thick and have been fabricated by UV LIGA. $d$ is the initial gap between both tools.

\section{Working principle of the tool changer}

To perform micromanipulation tasks of any kind of objects from 20 to $500 \mu \mathrm{m}$ in size, we have developed a microgripper (figure 1) that can be fixed on nearly any kind of microrobot (the suitable mechanical interface must be done to connect them together). In our case, the microgripper has been fixed on a X-Y-Z table actuated by linear stages (M-112 1DG from Physik Instrumente) for air application and by piezoelectric stick and slip actuators from the LSRO (Laboratoire des Systèmes RObotiques, Ecole Polytechnique Fédérale de Lausanne, Switzerland) for in SEM applications. The piezoelectric actuator of the microgripper has got four degrees of freedom that combine an in-plane ( $Y$ axis) and an out-of-plane motion ( $Z$ axis). At the tip of this actuator a pair of tools that are made of Nickel is fixed. The strokes of this microgripper are $+/-80 \mu \mathrm{m}$ along the $Y$ axis and $+/-200 \mu \mathrm{m}$ along the $\mathrm{Z}$ axis for $+/-100 \mathrm{~V}$ supply voltage. The blocking forces are $55 \mathrm{mN}$ and $10 \mathrm{mN}$ along $\mathrm{Y}$ and $\mathrm{Z}$ respectively. More details about this microgripper are given in ([13]).

Several kinds of pairs of tools have been designed with different tip shapes and different initial gaps between the tools $d$ (figure 2). The suitable pair of tools can be chosen depending on the size, shape and material of the object to manipulate.

To perform a microassembly sequence or micromanipulation in confined 


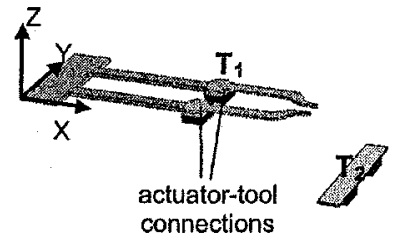

a)

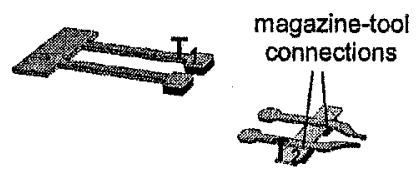

b)

Figure 3: Diagram of the actuator-tools-magazine-resistance set (a) in manipulation configuration, i.e. when a pair of tools is fixed at the tip of the actuator (b) in tool exchange configuration, i.e. when a pair of tools is fixed on the magazine.

spaces with flexibility, a tool changer adapted to the microgripper has been designed. This system allows to fix a pair of tools alternatively at the tip of the actuator or in a magazine (figure 3). Several pairs of tools are available in the magazine with different characteristics. The temporary fixation is possible due to the use of a thermal glue (Crystalbond 555-HMP belongs to Crystalbond TM series made by Aremco Products, Inc., USA) that is liquid at $65^{\circ} \mathrm{C}$ (in the air), $62{ }^{\circ} \mathrm{C}$ (in the vacuum) and solid at room temperature. Cycles of liquefaction and solidification can be performed without that the glue looses its properties. Very small amounts of glue (about $4 \mathrm{~nL}$ per contact) are placed at the contacts tools-actuator and tools-magazine (figure 4). Surface Mounted Devices resistances of $6 \Omega$ each have been placed under these contacts and can be heated up when supplied with a current (liquefaction of the glue). To solidify the glue, the supply of the resistances is switched off. Cycles of tool exchanges can be performed in air environment as well as in the vacuum chamber of a SEM. Figure 5 details the successive steps to do in order to perform a tool exchange. A user interface has been developed with Borland Builder $\mathrm{C}++$ to control both the microgripper and the tool changer allowing automatic tool exchanges for in air applications. Two minutes are necessary to exchange the pair of tools. This time is short compared to the one required to open the chamber of the SEM, change the gripper (for example), calibrate the new position of the tools, close the door of the SEM and obtain the required vacuum (15 minutes at least).

To improve the flexibility of the tool changer both resistances of the actuator can be supplied separately $\left(R_{1}\right.$ on figure 4$)$. Both resistances of each place of the magazine can also be supplied separately $\left(R_{2}\right.$ on figure 4$)$. By combination, it is so possible to fix one tool at the tip of the actuator whereas the second one is fixed on the magazine allowing the correction of the relative position between both tools (gap $d$ ). Figure 6 displays the successive steps to perform in order to change the gap between two tools.

This system is also used to set up the tools in the magazine. The first time, the tools are approximately placed in the magazine by hand using tweezers. The 


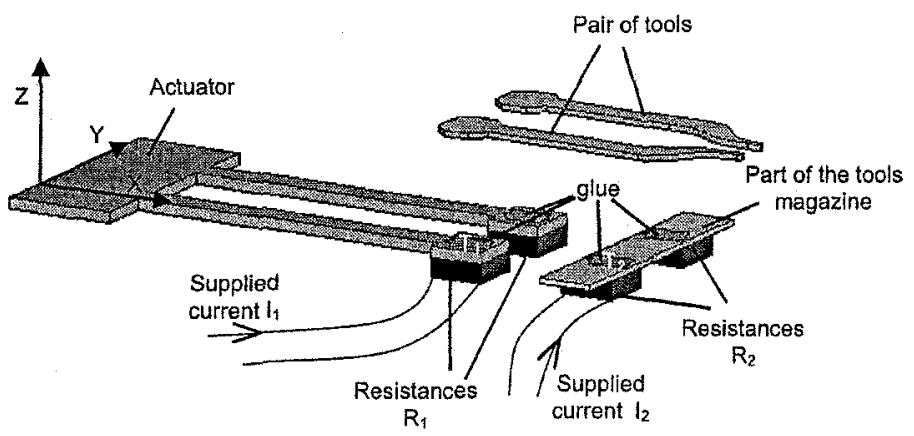

Figure 4: Diagram of the actuator-tools-magazine-resistances-glue set. Small amounts of glue are placed at the actuator-tools and tools-magazine contacts.
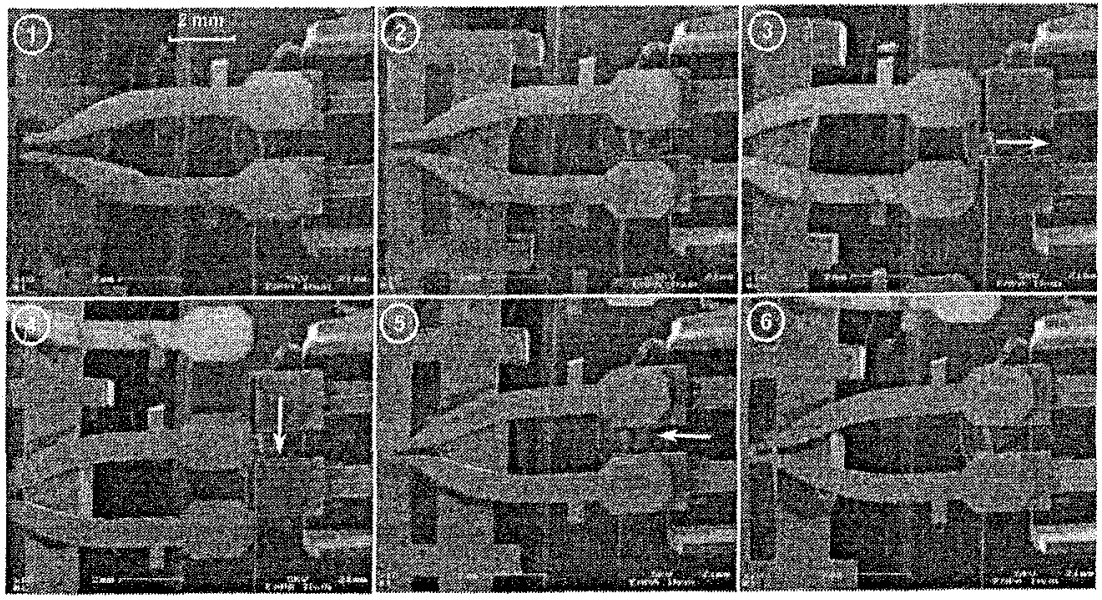

Figure 5: Successive steps for exchanging a pair of tools in the SEM: (1) a pair of tools is fixed at the tip of the actuator to perform the first micromanipulation task - (2) the first pair of tools is fixed both at the tip of the actuator and on the magazine - (3) the first pair of tools is released in the magazine - (4) the actuator alone reaches the position of the second pair of tools - (5) the second pair of tools is fixed both at the tip of the actuator and on the magazine - (6) the second pair of tools is fixed at the tip of the actuator to perform the second micromanipulation task. 


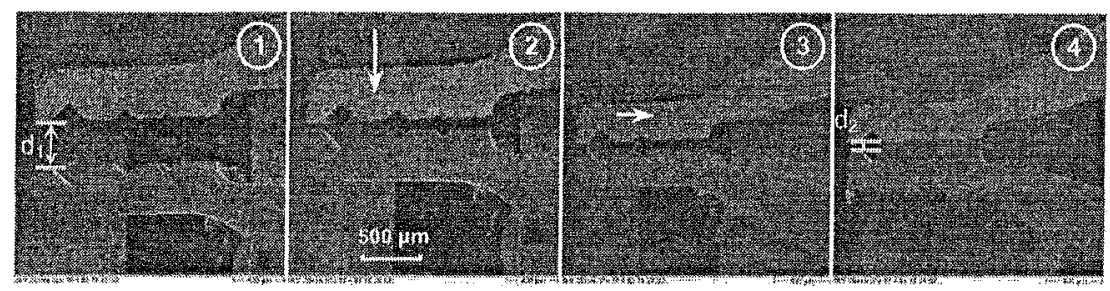

Figure 6: Successive steps to set up the relative position between both tools in the SEM. The initial gap between the tools is $d_{1}$ along the $Y$ axis. (1) One tool is fixed on the magazine (the one at the bottom) whereas the second tool is fixed at the tip of the actuator (the one at the top) - (2) motion in the Y direction to set the gap between the tools to $d_{2}-(3)$ correction of the relative position of the tools along the $X$ axis (if necessary) - (4) taking the pair of tools out of the magazine. The gap between the tools is now $d_{2}$.

relative position of the tools can then be corrected precisely using this system.

\section{Characterization of the tool changer}

Several studies have been performed to characterize the tool changer. First of all the tool positioning accuracy has been studied in air environment. Hundreds of cycles have been performed including one reference measurement, one measurement at the tip of one tool, the deposition of one tool, a displacement of the actuator alone, the removal of the same tool of the magazine. The measurements use a laser sensor (LC 2420 from Keyence) and are relative. The maximum positioning errors between two tool exchanges are $3.2 \mu \mathrm{m}, 2.3 \mu \mathrm{m}$ and $2.8 \mu \mathrm{m}$ along the $\mathrm{X}, \mathrm{Y}$ and $\mathrm{Z}$ axes respectively. The standard deviations are $0.73,0.47$ and $1.16 \mu \mathrm{m}$ and the averages are $0.74,0.62$ and $0.03 \mu \mathrm{m}$ along the $\mathrm{X}, \mathrm{Y}$ and $\mathrm{Z}$ axes respectively. When a deviation is too large, the relative position of the tools can be modified using the sequence defined in figure 6 .

The mechanical performances of the glue film have also been measured. These measurements showed that a force of $300 \mathrm{mN}$ has to be applied at the tip of one tool along the $\mathrm{Y}$ axis to break the film of glue between tool and actuator. $400 \mathrm{mN}$ are necessary to break the actuator and the blocking force of the actuator during micromanipulation tasks is $110 \mathrm{mN}$.. Thus, the film of glue acts as a fuse. As a consequence, micromanipulation tasks are performed safely.

Finally, during the liquefaction, the glue generates gas. This is not a problem in the air but can cause damage in the chamber of a SEM. Indeed, the pressure inside the chamber of a SEM must stay lower than $1.5 \times 10^{-5}$ millibars to allow a good working of the electron beam. So, the degassing process of the glue must be quantified to know whether it can prevent the good working of the electron 


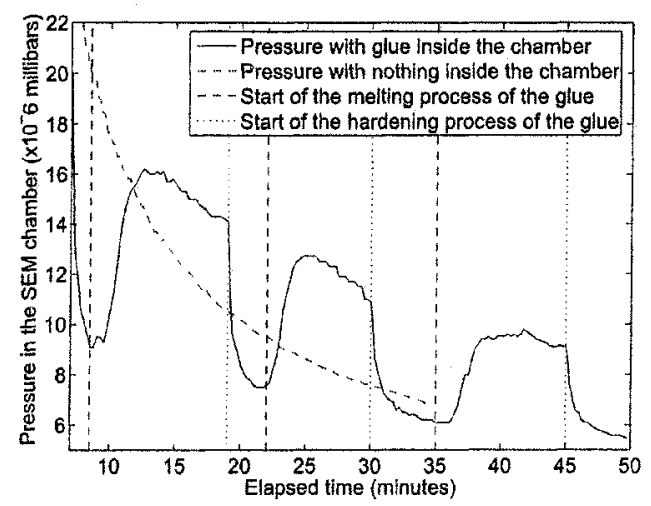

Figure 7: Pressure variation versus time during when a part of glue is successively liquefied and solidified several times inside the chamber of a SEM. This measurement can be compared to the pressure variation versus time given when there is nothing inside the chamber of the SEM (reference).

beam by affecting the vacuum. Pressure measurements have been performed to compare the pressure evolution versus time when there is nothing inside the SEM chamber and when there is an amount of glue undergoing cycles of liquefaction and solidification. The results of these measurements are given in figure 7 showing that there is a degassing process but low enough to allow the good working of the electron beam of the SEM. More details about the caracterization of the tool changer are available in ([5]) and ([4]).

\section{Microassembly of a ball bearing using the tool changer}

The assembly of a ball bearing has been tested in the chamber of a SEM (High vacuum SEM, Carl Zeiss DSM 962) to demonstrate both the microgripper capabilities and the effectiveness of the tool changer in vacuum environment. The external diameter of this bearing measures $1.6 \mathrm{~mm}$ and the diameter of the balls of this bearing is $200 \mu \mathrm{m}$ (picture 8 ). Figure 9 shows the successive steps that were performed to assemble the bearing. Several kinds of pairs of tools were necessary requiring the use of the tool changer. The first pair of tools was used to manipulate the first three parts. These parts have different sizes, so once again, the tool changer was used to correct the gap between the tools. 


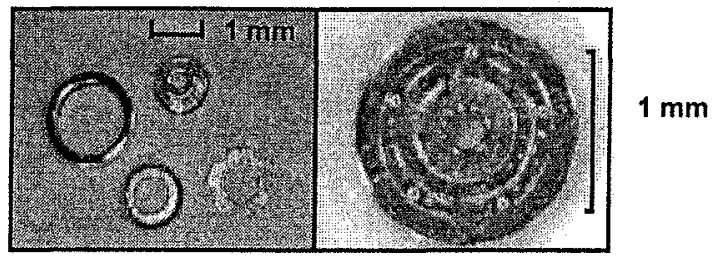

Figure 8: Five balls bearing before being assembled (left) and once assembled (right). The external ring measures $1.6 \mathrm{~mm}$ and the balls are $200 \mu \mathrm{m}$ in diameter.

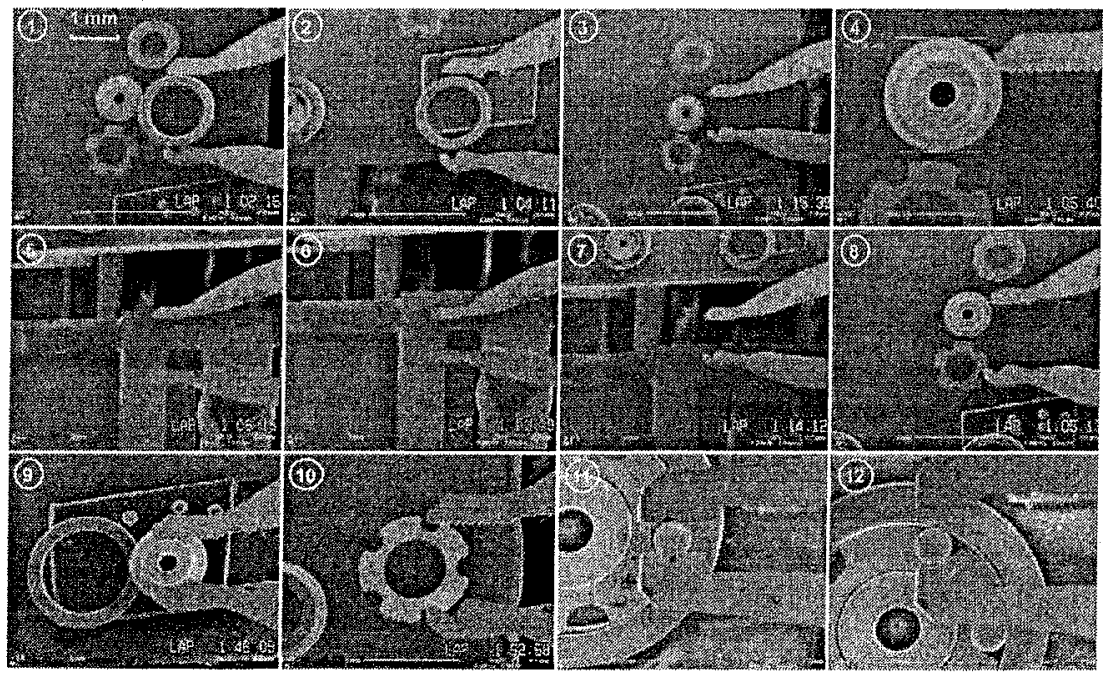

Figure 9: Assembly of a ball bearing in the SEM: sequence of elementary operations (1) taking of the external ring of the bearing. The gap between the tools measures $1.6 \mathrm{~mm}$ and corresponds to the diameter of the ring - (2) release of this ring on the workplane - (3) the gap between the tools is too large to take the axle of the bearing - (4) measurement of the size of the part to take - (5) reaching the magazine - (6) correction of the gap between the tools. This gap now measures $1 \mathrm{~mm}$ - (7) taking the pair of tools of the magazine - (8) approach to the second part to manipulate - (9) manipulation of the axle of the bearing (10) pick and place of the ball bearing casing using the same pair of tools and the same gap than before - (10-11) sequence of tool exchange to take a second pair of tools - (11) pick and place of the first ball - (12) pick and place of the second ball. 


\section{Conclusion}

To perform an assembly process or more generally micromanipulation tasks in air environment or inside the vacuum chamber of a SEM, we have designed a four degrees of freedom microgripper and a tool changer. This tool changer enables one to perform micromanipulation tasks using the pair of tools that is adapted to the object to manipulate. It is also possible to correct the relative position of the tools. Hundreds of automatic tool exchanges can be performed. The SEM compatibility of the tool changer has been successfully tested. This device brings flexibility and compactness to the micromanipulation cell in which it is used. As an example, the assembly of a ball bearing has been successfully performed in the vacuum chamber of a SEM. The principle of the tool changer is based on the use of a thermal glue and could be adapted on other kinds of microgripper or even on other kinds of devices.

\section{Acknowledgment}

The authors would like to thank the MPS company for the donation of microbearings, the AMiR institute (Oldenburg University, Germany) for the pressure measurements in their SEM and the LEO company (Oberkochen, Germany) for the thermal measurements inside their SEM. This work has notably been supported by the ROBOSEM project (European Project FP5 G1RD-CT200200675).

\section{References}

[1] K. F. Bohringer, R. S. Fearing, and K. Y. Goldberg. Handbook of industrial robotics. Wiley and sons, 1998. Chapter Microassembly.

[2] J. M. Breguet and A. Bergander. Toward the personal factory? SPIE, 4568:293-303, 2001.

[3] H. Van Brussel, J. Peirs, D. Reynaerts, A. Delchambre, G. Reinhart, N. Roth, M. Weck, and E. Zussman. Assembly of microsystems. Annals of the CIRP, 49(2):451-472, 2000.

[4] C. Clévy, A. Hubert, J. Agnus, and N. Chaillet. A micromanipulation cell including a tool changer. Journal of Micromechanics and Microengineering, 15:292-301, July 2005.

[5] C. Clévy, A. Hubert, and N. Chaillet. A new micro-tools exchange principle for micromanipulation. In IROS, Sendai, Japan, September 2004.

[6] R. Eberhardt, T. Scheller, G. Tittelbach, and V. Guyenot. Automated assembly of micro-optical components. SPIE, 3202:117-127, 1998. 
[7] S. Fatikow, A. Kortschack, H. Hudsen, T. Sievers, and T. Wich. Towards fully automated microhandling. In $I W M F$, pages 34-39, Shanghai, China, 2004.

[8] T. Kasaya, H. Miyazaki, S. Saito, and T. Sato. Micro object handling under sem by vision-based automatic control. In ICRA, pages 2189-2196, Detroit, USA, 1999.

[9] B. Kim, H. Kang, D.H. Kim, G.T. Park, and J.O. Park. Flexible microassembly system based on hybrid manipulation scheme. In International Conference on Intelligent Robots and Systems, pages 2091-2066, Las-Vegas, USA, October 2003.

[10] B.E. Kratochvil, K.B. Yesin, V. Hess, and B.J. Nelson. Design of a visually guided 6 dof micromanipulator system for 3d assembly of hybrid mems. In International Workshop on Microfactories, pages 128-133, Shanghai, China, 2004.

[11] H. Miyazaki and T. Sato. Mechanical assembly of three-dimensional microstructures from fine particles. Advanced robotics, 11(2):139-185, 1997.

[12] M. Nienhaus, W. Ehrfeld, F. Michel, V. Graeff, and A. Wolf. Handling and bonding of millimeterwave monolithic integrated circuits with high density interconnections for automotive and it applications. In 3rd workshop on "Area array packaging technologies", Berlin, Germany, 1999.

[13] R. Perez, J. Agnus, C. Clévy, A. Hubert, and N. Chaillet. Modelling, fabrication and validation of a high performance 2 dof microgripper. ASME/IEEE Transaction on Mechatronics, 10(2), April 2005.

[14] S. Saito, H. Miyazaki, and T. Sato. Pick and place operation of a micro object with high reliability and precision based on micro physics under sem. In ICRA, pages 2736-2743, Detroit Michigan, USA, May 1999.

[15] E. Shimada, J.A. Thompson, J. Yan, R. Wood, and R.S. Fearing. Prototyping millirobots using dextrous microassembly and folding. In $A S M E$ IMECE $/ D S C D$, pages 1-8, Orlando, USA, November 2000.

[16] G. D. Skidmore, M. Ellis, E. Parker, N. Sarkar, and R. Merkle. Micro assembly for top down nanotechnology. In Int symposium on Mechatronics and human science, pages 3-9, Nagoya, Japan, 2000.

[17] T. Tanikawa, M. Kawai, N. Koyachi, T. Arai, T. Ide, S. Kaneko, R. Ohta, and $\mathrm{T}$. Hirose. Force control system for autonomous micro manipulation. In ICRA, pages 610-615, Seoul, Korea, May 2001.

[18] J.A Thompson and R.S. Fearing. Automating microassembly with orthotweezers and force sensing. In IROS, Maui HI, 2001. 
[19] M. Weck and C. Peschke. Equipment technology for flexible and automated micro-assembly. Microsystem technologies, 10(3):241-246, 2004.

[20] B. Winzek, S. Schmitz, and T. Sterzl. Microgrippers with shape memory thin film actuators. In IPAS, pages 77-84, Bad Hofgastein, Austria, February 2004.

[21] G. Yang, J. A. Gaines, and B. J. Nelson. A flexible experimental workcell for efficient and reliable wafer-level $3 \mathrm{~d}$ microassembly. In ICRA, pages 133-138, Seoul, Korea, May 2001. 